doi:10.13108/2020-12-1-82

\title{
ALGEBRAICITY OF LATTICE OF $\tau$-CLOSED TOTALLY $\omega$-SATURATED FORMATIONS OF FINITE GROUPS
}

\author{
V.V. SHCHERBINA \\ Dedicated to a blessed memory of Leonid Alexandrovich Shemetkov
}

\begin{abstract}
All groups considered in this paper are assumed to be finite. The symbol $\omega$ denotes some nonempty set of primes, and $\tau$ is a subgroup functor in the sense of A.N. Skiba. We recall that a formation is a class of groups that is closed under taking homomorphic images and finite subdirect products. Functions of the form $f: \omega \cup\left\{\omega^{\prime}\right\} \rightarrow$ \{formations of groups are called $\omega$-local satellites (formation $\omega$-functions). Such functions are used to study the structure of $\omega$-saturated formations.

The paper is devoted to studying the properties of the lattice of all closed functorially totally partially saturated formations related to the algebraicity concept for a lattice of formations. We prove that for each subgroup functor $\tau$, the lattice $l_{\omega_{\infty}}^{\tau}$ of all $\tau$-closed totally $\omega$-saturated formations is algebraic. This generalizes the results by V.G. Safonov. In particular, we show that the lattice $l_{p_{\infty}}^{\tau}$ of all $\tau$-closed totally $p$-saturated formations is algebraic as well as the lattice $l_{\infty}^{\tau}$ of all $\tau$-closed totally saturated formations. Similar results are obtained for lattices of functorially closed totally partially saturated formations corresponding to certain subgroup functors $\tau$. Thus, we find new classes of algebraic lattices of formations of finite groups.
\end{abstract}

Keywords: formation of finite groups, totally $\omega$-saturated formation, lattice of formations, $\tau$-closed formation, algebraic lattice.

Mathematics Subject Classifcation: 20D10, 20F17

\section{INTRODUCTION}

All groups considered in this work are assumed to be finite. We follow a terminology used in works [1]- 4].

One of the most intensively developing directions in the formation theory is one aimed on studying internal structures of formations and their classifications. Universal tools of such studies are the methods and constructions of general lattice theory.

A modular property of the lattice of all formations established by A.N. Skiba [5], as well as of the lattice of all saturated formations gave an opportunity to employ lattice methods for solving many issues in the formation theory. The main results of the structural formation theory was exposed in monographs [1]-[3, [6]-[9]. Works [10]-[15] were devoted to studying a series of properties of the lattice of all totally saturated formations as well as a structural construction of totally saturated formations with prescribed restrictions on the lattice of of their totally saturated subformations.

V.V. Shcherbina, Algebraicity of lattice of $\tau$-Closed totally $\omega$-Saturated formations of FINITE GROUPS.

(c) Shcherbina V.V. 2020.

This research is supported by the Ministry of Education of Republic of Belorussia (SPSS "Konvergencia" 1.1.03.02).

Submitted April 12, 2019. 
A.N. Skiba [3] showed that for each integer $n$, the lattice of all $\tau$-closed $n$-mutliply saturated formations and the lattice of all soluble totally saturated formations are algebraic. A.N. Skiba and L.A. Shemetkov [16] proved the algebraicity of the lattice of $n$-mutliply $\mathfrak{L}$-composition formation. Later, I.P. Shabalina established the algebraicity of the lattice of $\tau$-closed $n$-mutliply $\omega$-saturated formations [17]. M.V. Zadorozhnyuk proved the algebraicity of the lattice of all $\tau$-closed solubly $\omega$-saturated formations [18]. The algebraicity of the lattice of all $\tau$-closed $n$ mutliply solubly $\omega$-saturated formations was proved by N.N. Vorob'ev and A.A. Tsarev [19], see also [8, Ch. 4, Thm. 4.6.12].

V.G. Safonov showed that the lattice of all $\tau$-closed totally saturated formations and the lattice of all totally $\omega$-saturated formations are algebraic [11, [20]. A series of properties of the lattice of totally partially saturated formations were studied in works [20]-[23].

In the present work we employ a functorial approach to prove that the lattice $l_{\omega_{\infty}}^{\tau}$ of all $\tau$-closed totally $\omega$-saturated formation is algebraic.

\section{Definitions And notations}

In what follows $\omega$ denotes some non-empty set of prime numbers and $\omega^{\prime}=\mathbb{P} \backslash \omega$. By symbols $F_{p}(G)$ and $O_{\pi}(G)$ we denote respectively the maximal normal $p$-nilpotent subgroup of group $G$ and the maximal normal $\pi$-subgroup of group $G$, while the symbol $\pi(G)$ stands for the set of all prime divisors of the group $G$. The symbols $\mathfrak{N}_{p}, \mathfrak{N}_{\pi}$ and $\mathfrak{S}_{\pi}$ denote the class of all $p$-groups, $\pi$-groups and soluble $\pi$-groups, respectively.

Following [4, by the symbol $G_{\omega d}$ we denote the maximal normal in $G$ subgroup $K$ with the property $\omega \cap \pi(H / N) \neq \varnothing$ for each composition factor $H / N$ in $K\left(G_{\omega d}=1\right.$ if $\omega \cap \pi(\operatorname{Soc}(G))=$ $\varnothing)$ ).

We recall that a formation is a class of groups closed with respect to taking homomorphic images and finite subdirect products.

Each function of form $f: \omega \cup\left\{\omega^{\prime}\right\} \rightarrow$ fformations of groups $\}$ is called $\omega$-local satellite (formation $\omega$-function). Following [4], to each $\omega$-local satellite $f$, we associate a class of groups

$$
\operatorname{LF}_{\omega}(f)=\left(G \mid G / G_{\omega d} \in f\left(\omega^{\prime}\right) \text { and } G / F_{p}(G) \in f(p) \text { for all } p \in \omega \cap \pi(G)\right) \text {. }
$$

If a formation $\mathfrak{F}$ is such that $\mathfrak{F}=\operatorname{LF}_{\omega}(f)$ for some $\omega$-local satellite $f$, then the formation $\mathfrak{F}$ is called $\omega$-local, and $f$ is called local satellite of this formation [4]. If all values of $f$ are located in $\mathfrak{F}$, then $f$ is called internal (or reduced) satellite.

A formation $\mathfrak{F}$ is called $\omega$-saturated if it contains each group $G$ satisfying the condition $G / L \in \mathfrak{F}$, where $L \subseteq \Phi(G) \cap O_{\omega}(G)$.

Let $A, B$ be groups, $\varphi: A \rightarrow B$ be an epimorphism, $\Omega$ and $\Sigma$ be some systems of subgroups in $A$ and $B$, respectively. Then by $\Omega^{\varphi}$ we denote the set $\left\{H^{\varphi} \mid H \in \Omega\right\}$, and by $\Sigma^{\varphi^{-1}}$ we denote the set $\left\{H^{\varphi^{-1}} \mid H \in \Sigma\right\}$ of all complete pre-images in $A$ of all groups in $\Sigma$.

Let $\mathfrak{X}$ be an arbitrary non-empty class of groups and to each group $G \in \mathfrak{X}$ some system of its subgroups $\tau(G)$ be associated. We say that $\tau$ is a subgroup $\mathfrak{X}$-functor in the sense of A.N. Skiba [3] (or, in other words, $\tau$ is a subgroup functor on $\mathfrak{X}$ ) if for each epimorphism $\varphi: A \rightarrow B$, where $A, B \in \mathfrak{X}$, the embeddings hold $(\tau(A))^{\varphi} \subseteq \tau(B),(\tau(B))^{\varphi^{-1}} \subseteq \tau(A)$ and for each group $G \in \mathfrak{X}$ we have $G \in \tau(G)$. If $\mathfrak{X}=\mathfrak{G}$ is the class of all groups, then the symbol $\mathfrak{X}$ is omitted and one says just a subgroup functor. By $\mathrm{S}(G)$ we denote the set of all subgroups of a group $G$, while $\mathrm{S}_{n}(G)$ is the set of all normal subgroups of the group $G$. A subgroup functor $\tau$ is called trivial if $\tau(G)=\{G\}$, and is called unit if $\tau(G)=\mathrm{S}(G)$ for each group $G$. The class of groups $\mathfrak{F}$ is called $\tau$-closed if $\tau(G) \subseteq \mathfrak{F}$ for each group $G \in \mathfrak{F}$. 
According the concept of multiple locality proposed by A.N. Skiba [24], see also [4], each formation is supposed to be 0 -mulitple $\omega$-local, while for $n \geqslant 1$, a formation $\mathfrak{F}$ is called $n$ multiple $\omega$-local if $\mathfrak{F}=\mathrm{LF}_{\omega}(f)$, where all values of $f$ are $(n-1)$-mutliply $\omega$-local formations. A formation $\mathfrak{F}$ is called totally $\omega$-local if it is $n$-mulitple $\omega$-local for all $n$. If at that a formation $\mathfrak{F}$ is $\tau$-closed, then $\mathfrak{F}$ is called $\tau$-closed $n$-mutliply $\omega$-local and respectively $\tau$-closed totally $\omega$-local.

In view of Theorem 1 in work [4], a formation $\mathfrak{F}$ is called $\omega$-local if and only if it is $\omega$-saturated. This is why $n$-mutliply $\omega$-local and totally $\omega$-local formations are also $n$-mutliply $\omega$-saturated and, respectively, totally $\omega$-saturated.

We recall that a lattice is a partially ordered set $L$, in which each two elements have an infimum denoted by $x \wedge y$ and the supremum denoted by $x \vee y$ [25, Ch. I, Sect. 4]. A lattice $L$ is called complete if each its subset $X$ have supremum and infimum in $L$. An element $a$ of the lattice $L$ is called compact if it follows from $a \leqslant \vee\left(x_{i} \mid i \in I\right)$ that $a \leqslant \vee\left(x_{i} \mid i \in J\right)$ for some finite subset $J \subset I$. A lattice $L$ is called algebraic if each its element $a \in L$ is the union of compact elements in the lattice $L$ [25, Ch. VIII, Sect. 5].

A non-empty system of formations $\theta$ is called complete lattice of formations if the intersection of each set of formations in $\theta$ again belongs to $\theta$ and the set $\theta$ contains a formation $\mathfrak{F}$, such that $\mathfrak{H} \subseteq \mathfrak{F}$ for each formation $\mathfrak{H} \in \theta$. Each complete lattice of formations is a complete lattice in the usual sense. A formation in $\theta$ is called $\theta$-formations. A satellite $f$ is called $\theta$-valued if all its values belong to $\theta$. The symbol $\theta^{\omega}$ denotes the set of all formations possessing a $\omega$-local $\theta$-valued satellite.

By $l_{\omega_{\infty}}^{\tau}$ we denote the set of all $\tau$-closed totally $\omega$-saturated formations. For each set of groups $\mathfrak{X}$ by $l_{\omega_{\infty}}^{\tau}$ form $\mathfrak{X}$ we denote the intersection of all $\tau$-closed totally $\omega$-saturated formations containing $\mathfrak{X}$. A formation $l_{\omega_{\infty}}^{\tau}$ form $\mathfrak{X}$ is called $\tau$-closed totally $\omega$-saturated formation generated by the set of groups $\mathfrak{X}$. If $\mathfrak{X}=\{G\}$, then $l_{\omega_{\infty}}^{\tau}$ form $\mathfrak{X}=l_{\omega_{\infty}}^{\tau}$ form $G$ is called one-generated $\tau$ closed totally $\omega$-saturated formation.

For all $\tau$-closed totally $\omega$-saturated formations $\mathfrak{M}$ and $\mathfrak{H}$ we let $\mathfrak{M} \vee_{\omega_{\infty}}^{\tau} \mathfrak{H}=l_{\omega_{\infty}}^{\tau}$ form $(\mathfrak{M} \cup \mathfrak{H})$ With respect to the operations $\vee_{\omega_{\infty}}^{\tau}$ and $\cap$, the set of all $\tau$-closed totally $\omega$-saturated formations $l_{\omega_{\infty}}^{\tau}$ partially ordered with respect to the inclusion $\subseteq$ is a complete lattice of formations, see [8, Ch. 1, Thm. 1.5.4]. In this lattice $\vee_{\omega_{\infty}}^{\tau}\left(\mathfrak{F}_{i} \mid i \in I\right)=l_{\omega_{\infty}}^{\tau}$ form $\left(\cup_{i \in I} \mathfrak{F}_{i}\right)$ and $\underset{i \in I}{\cap} \mathfrak{F}_{i}$ are respectively supremum and infimum for the subset $\left\{\mathfrak{F}_{i} \mid i \in I\right\}$ in $l_{\omega_{\infty}}^{\tau}$.

An $\omega$-local satellite, whose values are $l_{\omega_{\infty}}^{\tau}$-formation is called $l_{\omega_{\infty}}^{\tau}$-valued satellite.

Let $\left\{f_{i} \mid i \in I\right\}$ be some system of $l_{\omega_{\infty}}^{\tau}$-satellites. Then by $\vee_{\omega_{\infty}}^{\tau}\left(f_{i} \mid i \in I\right)$ we denote a satellite $f$ such that $f(a)=l_{\omega_{\infty}}^{\tau}$ form $\left(\cup_{i \in I} f_{i}(a)\right)$ for all $a \in \omega \cup\left\{\omega^{\prime}\right\}$ if for at least one of the formation we have $f_{i}(a) \neq \varnothing$. Otherwise we let $f(a)=\varnothing$.

For each set of groups $\mathfrak{X}$ we let $\mathfrak{X}\left(F_{p}\right)=$ form $\left(G / F_{p}(G) \mid G \in \mathfrak{X}\right)$ if $p \in \pi(\mathfrak{X})$ and $\mathfrak{X}\left(F_{p}\right)=\varnothing$ if $p \notin \pi(\mathfrak{X})$.

For an arbitrary $\tau$-closed totally $\omega$-saturated formation $\mathfrak{F}$, by $\mathfrak{F}_{\omega_{\infty}}^{\tau}$ we denote its minimal $\omega$-local $l_{\omega_{\infty}}^{\tau}$-valued satellite, that is, the intersection of all its $\omega$-local $l_{\omega_{\infty}}^{\tau}$-valued satellites of the formation $\mathfrak{F}$.

For an arbitrary totally $\omega$-saturated formation $\mathfrak{F}$, by $F$ we denote its canonical (maximal interior $\omega$-local) satellite. According Remark 1 in work [4], if $\mathfrak{F}=\mathrm{LF}_{\omega}(f)$ and $f$ is an arbitrary internal $\omega$-local satellite of the formation $\mathfrak{F}$, then the inequality holds: $f \leqslant F$.

\section{AUXILIARY RESUlts}

In order to prove the main results, we shall need some known facts of the formation theory of finite groups. 
Lemma 1 ([22, Lm. 3.2]). Let $\mathfrak{F}$ be a non-empty $\tau$-closed formation, $\pi$ be a set of prime numbers such that $\pi(\mathfrak{F}) \cap \omega \subseteq \pi$. Then the product $\mathfrak{S}_{\pi} \mathfrak{F}$ is a $\tau$-closed totally $\omega$-saturated formation.

Lemma 2 ([3, Ch. 2, Lm. 2.1.6]). Let A be a monolithic group with a non-Abelian monolith, $\mathfrak{M}$ be some $\tau$-clolsed semi-formation. And let $A \in l_{n}^{\tau}$ form $\mathfrak{M}$. Then $A \in \mathfrak{M}$.

Lemma 3 ([3, Ch. 4, Sect. 4.4]). The lattice $l_{n}^{\tau}$ is algebraic.

Lemma 4 ([23, Lm. 20]). Let $f_{i}$ be a $\omega$-local minimal $l_{\omega_{\infty}}^{\tau}$-valued satellite of a $\tau$-closed totally $\omega$-saturated formation $\mathfrak{F}_{i}$, where $i \in I$. Then $\vee_{\omega_{\infty}}^{\tau}\left(f_{i} \mid i \in I\right)$ is a minimal $l_{\omega_{\infty}}^{\tau}$-valued $\omega$-local satellite of the formation $\mathfrak{F}=\vee_{\omega_{\infty}}^{\tau}\left(\mathfrak{F}_{i} \mid i \in I\right)$.

Lemma 5 ([4, Lm. 4]). If $\mathfrak{F}=\operatorname{LF}_{\omega}(f)$ and $G / O_{p}(G) \in \mathfrak{F} \cap f(p)$ for some $p \in \omega$, then $G \in \mathfrak{F}$.

\section{MAIN RESULT}

Lemma 6. Let $\mathfrak{H}=l_{\omega_{\infty}}^{\tau}$ form $\left(\cup_{i \in I} \mathfrak{F}_{i}\right)$, where $\mathfrak{F}_{i}$ is a $\tau$-closed totally $\omega$-saturated formation $(i \in I), A \in \mathfrak{H}$ is a monolithic group. Then if $\operatorname{Soc}(A)$ is a non-Abelian group, then $A \in \cup_{i \in I} \mathfrak{F}_{i}$.

Proof. Let $A$ be a group satisfying the assumptions of the lemma, $\pi=\pi\left(\right.$ form $\left.\left(\cup_{i \in I} \mathfrak{F}_{i}\right)\right) \cap \omega$. According Lemma 1, $\mathfrak{S}_{\pi} \tau$ form $\left(\cup_{i \in I} \mathfrak{F}_{i}\right) \in l_{\omega_{\infty}}^{\tau}$. This is why

$$
l_{\omega_{\infty}}^{\tau} \text { form }\left(\cup_{i \in I} \mathfrak{F}_{i}\right) \subseteq \mathfrak{S}_{\pi} \tau \text { form }\left(\cup_{i \in I} \mathfrak{F}_{i}\right)
$$

Hence, $A \in \mathfrak{S}_{\pi} \tau$ form $\left(\cup_{i \in I} \mathfrak{F}_{i}\right)$. Since $\operatorname{Soc}(A)$ is a non-Abelian group, then $A \in \tau$ form $\left(\cup_{i \in I} \mathfrak{F}_{i}\right)$. Then in view of Lemma $2 A \in \cup_{i \in I} \mathfrak{F}_{i}$.

Theorem. The lattice $l_{\omega_{\infty}}^{\tau}$ of all $\tau$-closed totally $\omega$-saturated formations is algebraic.

Proof. Let us show first that for each group $A$, a one-generated $\tau$-closed totally $\omega$-saturated formation $\mathfrak{F}=l_{\omega_{\infty}}^{\tau}$ form $A$ is a compact element in the lattice $l_{\omega_{\infty}}^{\tau}$.

We assume a contrary. Then there exists a group $A$ and formations $\mathfrak{F}_{i} \in l_{\omega_{\infty}}^{\tau}, i \in I$, such that

$$
\mathfrak{F}=l_{\omega_{\infty}}^{\tau} \text { form } A \subseteq \mathfrak{H}=l_{\omega_{\infty}}^{\tau} \text { form }\left(\cup_{i \in I} \mathfrak{F}_{i}\right)
$$

and moreover,

$$
\mathfrak{F}=l_{\omega_{\infty}}^{\tau} \text { form } A \nsubseteq l_{\omega_{\infty}}^{\tau} \text { form }\left(\cup_{i \in J} \mathfrak{F}_{j}\right)
$$

for each finite subset $J \subset I$. Let $A$ be a group of a smallest order among the groups with such property. Let us show that the group $A$ is monolithic. Assume that $N_{1}$ and $N_{2}$ are two different minimal normal subgroups of the group $A$. Let $\mathfrak{L}=l_{\omega_{\infty}}^{\tau}$ form $\left(A / N_{1}\right), \mathfrak{M}=l_{\omega_{\infty}}^{\tau}$ form $\left(A / N_{2}\right)$.

Since $\left|A / N_{1}\right|<|A|$ and $\left|A / N_{2}\right|<|A|$, then in view of the choice of the group $A$, it follows from the inclusions

$$
\begin{aligned}
& \mathfrak{L}=l_{\omega_{\infty}}^{\tau} \text { form } A / N_{1} \subseteq \mathfrak{H}=l_{\omega_{\infty}}^{\tau} \text { form }\left(\cup_{i \in I} \mathfrak{F}_{i}\right) \\
& \mathfrak{M}=l_{\omega_{\infty}}^{\tau} \text { form } A / N_{2} \subseteq \mathfrak{H}=l_{\omega_{\infty}}^{\tau} \text { form }\left(\cup_{i \in I} \mathfrak{F}_{i}\right)
\end{aligned}
$$

that there exist the sets of indices $i_{1}, \ldots, i_{k}$ and $j_{1}, \ldots, j_{l}$, such that

$$
\begin{aligned}
& \mathfrak{L} \subseteq l_{\omega_{\infty}}^{\tau} \text { form }\left(\mathfrak{F}_{i_{1}} \cup \ldots \cup \mathfrak{F}_{i_{k}}\right), \\
& \mathfrak{M} \subseteq l_{\omega_{\infty}}^{\tau} \text { form }\left(\mathfrak{F}_{j_{1}} \cup \ldots \cup \mathfrak{F}_{j_{l}}\right) .
\end{aligned}
$$


Therefore,

$$
\mathfrak{F}=\mathfrak{L} \vee_{\omega_{\infty}}^{\tau} \mathfrak{M} \subseteq l_{\omega_{\infty}}^{\tau} \text { form }\left(\mathfrak{F}_{i_{1}} \cup \ldots \cup \mathfrak{F}_{i_{k}} \cup \mathfrak{F}_{j_{1}} \cup \ldots \cup \mathfrak{F}_{j_{l}}\right)
$$

We have obtained a contradiction and hence, $A$ is a monolithic group.

Let $P=\operatorname{Soc}(A)$. Assume that $P$ is a non-Abelian group. Since $A \in l_{\omega_{\infty}}^{\tau}$ form $\left(\underset{i \in I}{\cup} \mathfrak{F}_{i}\right)$, by Lemma 6 we get that $A \in \cup_{i \in I} \mathfrak{F}_{i}$. Then there exists $i_{0} \in I$ such that $A \in \mathfrak{F}_{i_{0}}$, which is a contradiction. Therefore, $P$ is an Abelian p-group. Let $\pi=\pi\left(\right.$ form $\left.\left(\cup_{i \in I} \mathfrak{F}_{i}\right)\right) \cap \omega$.

Hence,

Assume that $p \notin \omega$. Then $p \notin \pi$. In view of Lemma 1, we have $\mathfrak{S}_{\pi} \tau$ form $\left(\cup_{i \in I} \mathfrak{F}_{i}\right) \in l_{\omega_{\infty}}^{\tau}$.

Since

$$
\mathfrak{H}=l_{\omega_{\infty}}^{\tau} \text { form }\left(\cup_{i \in I} \mathfrak{F}_{i}\right) \subseteq \mathfrak{S}_{\pi} \tau \text { form }\left(\cup_{i \in I} \mathfrak{F}_{i}\right)
$$

then

$$
A \in \mathfrak{H}=l_{\omega_{\infty}}^{\tau} \text { form }\left(\cup_{i \in I}^{\cup} \mathfrak{F}_{i}\right)
$$

Since $p \notin \pi$, then

$$
A \in \mathfrak{S}_{\pi} \tau \text { form }\left(\cup_{i \in I} \mathfrak{F}_{i}\right)
$$

$$
A \in \tau \text { form }\left(\cup_{i \in I}^{\cup} \mathfrak{F}_{i}\right) .
$$

Hence, according Lemma 3, there exist indices $i_{1}, \ldots, i_{m}$, such that

$$
\tau \text { form } A \subseteq \tau \text { form }\left(\mathfrak{F}_{i_{1}} \cup \ldots \cup \mathfrak{F}_{i_{m}}\right) .
$$

The inclusion

$$
\tau \text { form }\left(\mathfrak{F}_{i_{1}} \cup \ldots \cup \mathfrak{F}_{i_{m}}\right) \subseteq l_{\omega_{\infty}}^{\tau} \text { form }\left(\mathfrak{F}_{i_{1}} \cup \ldots \cup \mathfrak{F}_{i_{m}}\right)
$$

yield that

$$
\tau \text { form } A \subseteq l_{\omega_{\infty}}^{\tau} \text { form }\left(\mathfrak{F}_{i_{1}} \cup \ldots \cup \mathfrak{F}_{i_{m}}\right)
$$

Therefore,

$$
l_{\omega_{\infty}}^{\tau} \text { form } A \subseteq l_{\omega_{\infty}}^{\tau} \text { form }\left(\mathfrak{F}_{i_{1}} \cup \ldots \cup \mathfrak{F}_{i_{m}}\right) \text {, }
$$

which is a contradiction. This is why $p \in \omega$. Since

$$
l_{\omega_{\infty}}^{\tau} \text { form }\left(A / \Phi(A) \cap O_{\omega}(A)\right)=l_{\omega_{\infty}}^{\tau} \text { form } A,
$$

by the choice of the group $A$, we have $P \nsubseteq \Phi(A)$. This is why $P=C_{A}(P)=F_{p}(A)=F(A)=$ $O_{p}(A)$ and $A=[P] B$, where $B$ is some maximal subgroup in $A$. Let $f_{i}, f, h$ be minimal $l_{\omega_{\infty}}^{\tau}$-valued $\omega$-local satellites of the formations $\mathfrak{F}_{i}, \mathfrak{F}$ and $\mathfrak{H}$, respectively. Then by Lemma 4

$$
h=\vee_{\omega_{\infty}}^{\tau}\left(f_{i} \mid i \in I\right) .
$$

Since $P=F_{p}(A)$ and $A \in \mathfrak{H}$, then

$$
B \cong A / F_{p}(A) \in h(p)=\vee_{\omega_{\infty}}^{\tau}\left(f_{i}(p) \mid i \in I\right) .
$$

Since $|B|<|A|$, the choice of the group $A$ ensures that there exists a set of indices $J=$ $\left\{j_{1}, \ldots, j_{n}\right\}$ such that

$$
B \cong A / F_{p}(A) \in \vee_{\omega_{\infty}}^{\tau}\left(f_{j}(p) \mid j \in J\right) .
$$

According Lemma 4, $l=\vee_{\omega_{\infty}}^{\tau}\left(f_{j}(p) \mid j \in J\right)$ is a minimal $l_{\omega_{\infty}}^{\tau}$-valued satellite of the formation $\mathfrak{L}=\vee_{\omega_{\infty}}^{\tau}\left(\mathfrak{F}_{j} \mid j \in J\right)$. Therefore,

$$
A / O_{p}(A) \cong B \in l(p)=\mathrm{V}_{\omega_{\infty}}^{\tau}\left(f_{j}(p) \mid j \in J\right) .
$$

In view of Lemma 5 , we have $A \in \mathfrak{L}$. Therefore,

$$
\mathfrak{F}=l_{\omega_{\infty}}^{\tau} \text { form } A \subseteq \mathfrak{L}=\vee_{\omega_{\infty}}^{\tau}\left(\mathfrak{F}_{j} \mid j \in J\right)
$$


The obtained contradiction shows that $\mathfrak{F}$ is a compact element in the lattice $l_{\omega_{\infty}}^{\tau}$. Since each $\tau$-closed totally $\omega$-saturated formation is the union of its one-generated $\tau$-closed totally $\omega$ saturated subformations in the lattice $l_{\omega_{\infty}}^{\tau}$, the lattice $l_{\omega_{\infty}}^{\tau}$ is algebraic.

We mention the main corollaries of the proven theorem.

If $\tau$ is a trivial subgroup functor, by this theorem we obtain the following corollary.

Corollary 1 ([20, V.G. Safonov]). The lattice $l_{\infty}^{\omega}$ of all totally $\omega$-saturated formations is algebraic.

If $\omega=\mathbb{P}$, the theorem implies the following corollary.

Corollary 2 ([11, V.G. Safonov]). The lattice $l_{\infty}^{\tau}$ of all $\tau$-closed totally saturated formations is algebraic.

As $\omega=\mathbb{P}$, for the trivial subgroup functor the theorem gives the following corollary.

Corollary 3 ([11, V.G. Safonov]). The lattice $l_{\infty}$ of all totally saturated formations is algebraic.

If $\omega=\{p\}$, by the theorem we obtain the following corollary.

Corollary 4. The lattice $l_{p_{\infty}}^{\tau}$ of all $\tau$-closed totally p-saturated formations is algebraic.

For the unit subgroup functor Corollary 4 implies the following statement.

Corollary 5. The lattice of all hereditary totally p-saturated formations is algebraic.

In the case when $\tau(G)=\mathrm{S}_{n}(G)$ for each group $G$, by Corollary 4 we obtain the following statement.

Corollary 6. The lattice of all normally hereditary totally p-saturated formations is algebraic.

\section{General REMARKS}

We recall that a sublattice of a lattice $L$ is a subset $H \subseteq L$ such that iuf $a \in H, b \in H$, then $a \wedge b \in H$ and $a \vee b \in H$ [25, Ch. I, Sect. 4]. A sublattice of a lattice is again a lattice with the same operations of union and intersections. We also recall that a sublattice $H$ of a complete lattice $L$ is called complete if for each non-empty subset $X \subseteq H$ the belonging $\sup _{L} X \in H$ holds and $\inf _{L} X \in H$ [26, Ch. V, Sect. 1.2]. In this case, the identities $\sup _{H} X=\sup _{L} X$ and $\inf _{H} X=\inf _{L} X$ hold. It is easy show that a complete sublattice of an algebraic lattice is an algebraic lattice. As it follows from a known result by Ph.M. Whitman, see [27, Thm. 2] and also [26, Ch. V, Sect. 5.1], an arbitrary sublattice of an algebraic lattice is not necessary an algebraic lattice.

As it was mentioned above, the lattice $l_{\omega_{n}}^{\tau}$ is algebraic, see [17]. At the same time, the lattice $l_{\omega_{\infty}}^{\tau}$ is not a complete sublattice in $l_{\omega_{n}}^{\tau}$. Moreover, we shall show that for an arbitrary set of prime numbers $\omega$ such that $|\omega|>1$ and for an arbitrary non-negative integer $n$, the lattice $l_{\omega_{\infty}}^{\tau}$ is not a sublattice in $l_{\omega_{n}}^{\tau}$.

It is sufficient to show that the lattice of soluble $l_{\omega_{\infty}}^{\tau}$-formations is not a sublattice in $l_{\omega_{n}}^{\tau}$. We employ a method by A.N. Skiba, see [3, Ch.4, Sect. 4.1] and [8, Ch. 4, Sect. 4.5], and also work [28]).

We argue by the induction in $n$. Let $n=0$. We consider a formation $\mathfrak{F}=\mathfrak{N}_{p} \mathfrak{N}_{r} \vee \mathfrak{N}_{p} \mathfrak{N}_{q}$, where $p \in \omega, p, r$ and $q$ are mutually different prime numbers. Since the formations $\mathfrak{N}_{p}, \mathfrak{N}_{r}$ and $\mathfrak{N}_{q}$ are s-closed and totally saturated, they are also $\tau$-closed totally $\omega$-saturated. Then according Lemma 11 in [23], the formations $\mathfrak{N}_{p} \mathfrak{N}_{r}$ and $\mathfrak{N}_{p} \mathfrak{N}_{q}$ are also $\tau$-closed totally $\omega$-saturated. We 
also observe that they are soluble and moreover, $\mathfrak{N}_{p} \mathfrak{N}_{r} \subset \mathfrak{N}_{\omega}^{1} \mathfrak{N}_{r}=\mathfrak{N}_{\omega} \mathfrak{N}_{r}$ and $\mathfrak{N}_{p} \mathfrak{N}_{q} \subset \mathfrak{N}_{\omega}^{1} \mathfrak{N}_{q}=$ $\mathfrak{N}_{\omega} \mathfrak{N}_{q}$.

Let us show that the formation $\mathfrak{F}$ is not $\omega$-saturated. Assume that this is not true. Let $f$ be a minimal $\omega$-local satellite of the formation $\mathfrak{F}$. Then $f(p)=\mathfrak{N}_{\{r, q\}}$. Let $Z_{r}$ and $Z_{q}$ be some groups of orders $r$ and $q$, respectively. In view of Corollary 10.7 in [2, Ch. B] the group $B=Z_{r} \times Z_{q}$ possesses a prime exact module $P$ over the field $\mathbb{F}_{p}$. Let $G=[P] B$. Then in view of Lemma 5, $G \in \mathfrak{F}$. It is easy to see that $G \notin \mathfrak{N}_{p} \mathfrak{N}_{r} \cup \mathfrak{N}_{p} \mathfrak{N}_{q}$. Hence, according Corollary 1.2.26 in [3, Ch. 1], in $\mathfrak{F}$ there exists a group $H$ with normal sugroups $N, M, N_{1}, \ldots, N_{t} ; M_{1}, \ldots$, $M_{t}, t \geqslant 2$, such that the following statements hold:

1) $H / N \cong G, M / N=\operatorname{Soc}(H / N)$;

2) $N_{1} \cap \ldots \cap N_{t}=1$

3) $H / N_{i}$ is a monolithic $\mathfrak{N}_{p} \mathfrak{N}_{r} \cup \mathfrak{N}_{p} \mathfrak{N}_{q}$-group with a monolith $M_{i} / N_{i}$, which is $H$-isomorphic to $M / N$. Let $H / N_{1} \in \mathfrak{N}_{p} \mathfrak{N}_{r}$. Since $C_{G}(P)=P$, then $M=C_{H}(M / N)$. Hence, $M_{1} \subseteq M$ and $B=Z_{r} \times Z_{q} \in \mathfrak{N}_{p} \mathfrak{N}_{r}$. The obtained contradiction shows that the formation $\mathfrak{F}$ is not $\omega$-saturated. Hence, $\mathfrak{F}$ is not totally $\omega$-saturated. In view of Corollary 1.2.24 in [3, Ch. 1], we have:

$$
\begin{aligned}
& \mathfrak{N}_{p} \mathfrak{N}_{r} \vee_{\omega_{0}}^{\tau} \mathfrak{N}_{p} \mathfrak{N}_{q}=\tau \text { form }\left(\mathfrak{N}_{p} \mathfrak{N}_{r} \cup \mathfrak{N}_{p} \mathfrak{N}_{q}\right)= \\
& \quad \text { form }\left(\mathfrak{N}_{p} \mathfrak{N}_{r} \cup \mathfrak{N}_{p} \mathfrak{N}_{q}\right)=\mathfrak{N}_{p} \mathfrak{N}_{r} \vee \mathfrak{N}_{p} \mathfrak{N}_{q}
\end{aligned}
$$

Therefore, the lattice of soluble $l_{\omega_{\infty}}^{\tau}$-formations in not a sublattice in $l_{\omega_{0}}^{\tau}$.

Let $n>1$ and the statement be true for $n-1$. Then there exist solvable $l_{\omega_{\infty}}^{\tau}$-formation $\mathfrak{M} \subset \mathfrak{N}_{\omega}^{n} \mathfrak{N}_{r}$ and $\mathfrak{H} \subset \mathfrak{N}_{\omega}^{n} \mathfrak{N}_{q}$ such that $\mathfrak{M} \vee_{\omega_{n-1}}^{\tau} \mathfrak{H} \notin l_{\omega_{\infty}}^{\tau}$. Let $\mathfrak{M}_{1}=\mathfrak{N}_{\omega} \mathfrak{M}, \mathfrak{H}_{1}=\mathfrak{N}_{\omega} \mathfrak{H}$. In view of Lemma 4.5.2 in [8, Ch. 4], the formations $\mathfrak{M}_{1}$ and $\mathfrak{H}_{1}$ have such internal $l_{\omega_{\infty}}^{\tau}$-valued $\omega$-local satellites $m$ and $h$, respectively, such that for each $a \in \omega \cup\left\{\omega^{\prime}\right\}$ the identities $m(a)=\mathfrak{M}$, $h(a)=\mathfrak{H}$ hold. Hence, both formations belong to the lattice $l_{\omega_{\infty}}^{\tau}$. We note that the formations $\mathfrak{M}_{1}$ and $\mathfrak{H}_{1}$ are soluble. Moreover, taking into account that $\mathfrak{M} \subset \mathfrak{N}_{\omega}^{n} \mathfrak{N}_{r}$ and $\mathfrak{H} \subset \mathfrak{N}_{\omega}^{n} \mathfrak{N}_{q}$, we have:

$$
\mathfrak{M}_{1}=\mathfrak{N}_{\omega} \mathfrak{M} \subset \mathfrak{N}_{\omega}\left(\mathfrak{N}_{\omega}^{n} \mathfrak{N}_{r}\right)=\left(\mathfrak{N}_{\omega} \mathfrak{N}_{\omega}^{n}\right) \mathfrak{N}_{r}=\mathfrak{N}_{\omega}^{n+1} \mathfrak{N}_{r}
$$

and

$$
\mathfrak{H}_{1}=\mathfrak{N}_{\omega} \mathfrak{M} \subset \mathfrak{N}_{\omega}\left(\mathfrak{N}_{\omega}^{n} \mathfrak{N}_{q}\right)=\left(\mathfrak{N}_{\omega} \mathfrak{N}_{\omega}^{n}\right) \mathfrak{N}_{q}=\mathfrak{N}_{\omega}^{n+1} \mathfrak{N}_{q} .
$$

Suppose that $\mathfrak{M}_{1} \vee_{\omega_{n}}^{\tau} \mathfrak{H}_{1} \in l_{\omega_{\infty}}^{\tau}$. Since according Lemma 4.5.4 in [8, Ch. 4]

$$
\mathfrak{M}_{1} \vee_{\omega_{n}}^{\tau} \mathfrak{H}_{1}=\mathfrak{N}_{\omega} \mathfrak{M} \vee_{\omega_{n}}^{\tau} \mathfrak{N}_{\omega} \mathfrak{H}=\mathfrak{N}_{\omega}\left(\mathfrak{M} \vee_{\omega_{n-1}}^{\tau} \mathfrak{H}\right)
$$

and

$$
\begin{aligned}
\mathfrak{M}_{1} \vee_{\omega_{n}}^{\tau} \mathfrak{H}_{1} & =l_{\omega_{n}}^{\tau} \text { form }\left(\mathfrak{M}_{1} \cup \mathfrak{H}_{1}\right) \\
& =l_{\omega_{\infty}}^{\tau} \text { form }\left(\mathfrak{M}_{1} \cup \mathfrak{H}_{1}\right)=\mathfrak{M}_{1} \vee_{\omega_{\infty}}^{\tau} \mathfrak{H}_{1}
\end{aligned}
$$

by Lemma 4.5.5 in [8], the formation $\mathfrak{M} \vee_{\omega_{n-1}}^{\tau} \mathfrak{H}$ is totally $\omega$-saturated. Therefore, $\mathfrak{M} \vee_{\omega_{n-1}}^{\tau} \mathfrak{H} \in$ $l_{\omega_{\infty}}^{\tau}$, that contradicts the choice of the formations $\mathfrak{M}$ and $\mathfrak{H}$. Hence, the lattice of the soluble, and hence of all $l_{\omega_{\infty}}^{\tau}$-formations is not a sublattice in $l_{\omega_{n}}^{\tau}$.

Thus, the algebraicity, as any other property of the lattice $l_{\omega_{\infty}}^{\tau}$ is not in general an implication of the algebraicity or the corresponding property of the lattice $l_{\omega_{n}}^{\tau}$, see also Sections 4.2 and 4.4 in Ch. 4 of the monograph by A.N. Skiba [3]. In view of this we mention that the above given proof of the algebraicity of the lattice $l_{\omega_{\infty}}^{\tau}$ of all $\tau$-closed totally $\omega$-saturated formations base essentially on the properties of the lattice $l_{\omega_{\infty}}^{\tau}$ established earlier in works by V.G. Safonov and I.N. Safonov [22] and in the work by the author and V.G. Safonov [23]. It does not employ the results of work by I.P. Shabalina [17] and they are not implied by these results. 
We finally observe that similar remarks are true also for the theory of soluble $\omega$-saturated formations. Arguing as above, one can show that under the same assumptions for the set of prime numbers $\omega$ and an integer number $n(|\omega|>1, n \geqslant 0)$, the lattice $c_{\omega_{\infty}}^{\tau}$ of all $\tau$-closed totally soluble $\omega$-saturated formations is not a sublattice of the lattice $c_{\omega_{n}}^{\tau}$ of all $\tau$-closed $n$-mutliply solubly $\omega$-saturated formations.

In conclusion we mention that in a recently published work [29], A.A. Tsarev established the algebraicity of the lattice $c_{\infty}$ of all totally composite formations. The algebraicity of the lattice $c_{n}$ of all $n$-mutliply composite formations is implied by the algebraicity of the lattice $c_{\omega_{n}}^{\tau}$ for a trivial subgroup functor $\tau$ and $\omega=\mathbb{P}$, which was obtained earlier in a joint work of N.N. Vorob'ev and A.A. Tsarev [19], see also [8, Ch. 4, Thm. 4.6.12].

The author expresses his deep gratitude to the referee for useful remarks allowed to improve the paper.

\section{BIBLIOGRAPHY}

1. L.A. Shemetkov, A.N. Skiba. Formations of algebraic systems. Nauka, Moscow (1989). (in Russian).

2. K. Doerk, T. Hawkes. Finite soluble groups. Walter de Gruyter, Berlin (1992).

3. A.N. Skiba. Algebra of formations, Belarusskaya Navuka, Minsk (1997). (in Russian).

4. A.N. Skiba, L.A. Shemetkov. Multiply $\omega$-local formations and Fitting classes of finite groups // Matem. Trudy. 2:2, 114-147 (1999). (in Russian).

5. A.N. Skiba. On local formations of length $5 / /$ in "Arithmetic and subgroup structure of finite groups", Proc. of Gomel seminar. Nauka Tekh., Minsk 135-149 (1986). (in Russian).

6. W. Guo. The theory of classes of groups. Kluwer Academic Publishers, Beijing (2000).

7. A. Ballester-Bolinches, L.M. Ezquerro. Classes of finite groups. Springer, Dordrecht (2006).

8. N.N. Vorob'ev. Algebra of classes of finite groups. Vitebsk State Univ. named after P.M. Masherov, Vitebsk (2012). (in Russian).

9. W. Guo. Structure theory for canonical classes of finite groups. Springer, Berlin (2015).

10. V.G. Safonov. On modularity of lattice of $\tau$-closed totally saturated formations of finite groups // Ukrain. Matem. Zhurn. 58:6, 852-858 (2006). [Ukr. Math. J. 58:6, 967-973 (2006).]

11. V.G. Safonov. The property of being algebraic for the lattice of all $\tau$-closed totally saturated formations // Algebra i Logika. 45:5, 620-626 (2006).[Algebra Logic. 45:5, 353-356 (2006).]

12. V.G. Safonov. Characterization of the soluble one-generator totally saturated formations of finite groups // Sibir. Matem. Zhurn. 48:1, 185-191 (2007). [Siber. Math. J. 48:1, 150-155 (2007).]

13. V.G. Safonov. On a question of the theory of totally saturated formations of finite groups // Algebra Colloq. 15:1, 119-128 (2008).

14. V.G. Safonov, L.A. Shemetkov. Sublattices of the lattice of totally saturated formations of finite groups // Dokl. Nats. Akad. Nauk Belarusi. 52:4, 34-37 (2008). (in Russian).

15. V.G. Safonov. $\mathfrak{G}$-separability of the lattice of $\tau$-closed totally saturated formations // Algebra $\mathrm{i}$ Logika. 49:5, 692-704 (2010). [Algebra Logic. 49:5, 470-479 (2010).]

16. A.N. Skiba, L.A. Shemetkov. Multiply $\mathfrak{L}$-composite formations of finite groups // Ukrain. Matem. Zhurn. 52:6, 783-797 (2000). [Ukrain. Math. J. 52:6, 898-913 (2000).]

17. I.P. Shabalina. Algebraicity of lattice of $\tau$-closed n-mutliply $\omega$-local formations // Izv. Gomel. Gosud. Univ. im. F. Skoriny. Voprosy algebry-18. 14:5, 59-67 (2002). (in Russian).

18. M.V. Zadorozhnyuk. On elements of height 3 in lattice of $\tau$-valued $\omega$-composition formations // Vestnik Grodnen. Gosud. Univ. im. Ya. Kupaly. Ser. 2. Matem. Fiz. Inform. Vychisl. Tekh. Uprav. 2, 16-21 (2008). (in Russian).

19. N.N. Vorob'ev, A.A. Tsarev. On the modularity of the lattice of $\tau$-closed n-multiply $\omega$-composite formations // Ukrain. Matem. Zhurn. 62:4, 453-463 (2010). [Ukrain. Math. J. 62:4, 518-529 (2010).]

20. V.G. Safonov. On totally $\omega$-saturated formations of finite groups // Preprint: Gomel. Gosud. Univ. im. F. Skoriny. 7, (2004). (in Russian). 
21. V.G. Safonov, I.N. Safonova. On minimal totally $\omega$-saturated non-nilpotent formations of finite groups // Vestnik Vitebsk. Gosud. Univ. 84:6, 9-15 (2014). (in Russian).

22. V.G. Safonov, I.N. Safonova. Separability of the lattice of $\tau$-closed totally $\omega$-saturated formations of finite groups // Probl. Fiz. Mat. Tekh. 33:4, 76-83 (2017). (in Russian).

23. V.V. Shcherbina, V.G. Safonov. On sublattices of lattice of partially totally saturated formations of finite groups // Nauchn. Vedom. Belgorod. Gosud. Univ. Matem. Fiz. 51:1, 64-87 (2019). (in Russian).

24. A.N. Skiba. Characterization of finite solvable groups of given nilpotent length // Vopr. Algebry 3, 21-31 (1987). (in Russian).

25. G. Birkhoff. Lattice theory. Amer. Math. Soc. Providence, R.I. (1967).

26. V.A. Artamonov, V.N. Salii, L.A. Skornyakov, L.N. Shevrin, E.G. Shul'geifer. General algebra. V. 2. Nauka, Moscow (1991). (in Russian).

27. Ph.M. Whitman. Lattices, equivalence relations, and subgroups // Bull. Amer. Math. Soc. 52:6, 507-522 (1946).

28. L.A. Shemetkov, A.N. Skiba, N.N. Vorob'ev. On lattices of formations of finite groups // Algebra Colloq.17:4, 557-564 (2010).

29. A.A. Tsarev. On the lattice of all totally composition formations of finite groups // Ricerche di Mat. 68, 693-698 (2019).

Vladimir Vladimirovich Shcherbina, Belorus State University,

Nezavisimosti av. 4, 220030, Minsk, Republic of Belorus

E-mail: shcherbinavv@tut.by 